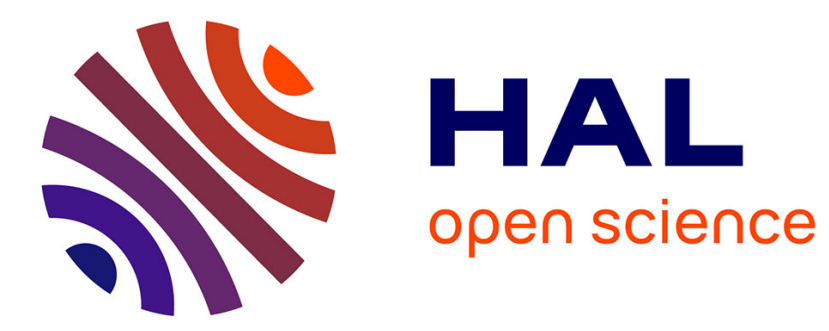

\title{
Penser la conception citoyenne de la culture de l'information
}

\author{
Olivier Le Deuff
}

\section{To cite this version:}

Olivier Le Deuff. Penser la conception citoyenne de la culture de l'information. Les Cahiers du numérique, 2009, 5 (3), pp.39-49. sic_00607084

\section{HAL Id: sic_00607084 \\ https://archivesic.ccsd.cnrs.fr/sic_00607084}

Submitted on 7 Jul 2011

HAL is a multi-disciplinary open access archive for the deposit and dissemination of scientific research documents, whether they are published or not. The documents may come from teaching and research institutions in France or abroad, or from public or private research centers.
L'archive ouverte pluridisciplinaire HAL, est destinée au dépôt et à la diffusion de documents scientifiques de niveau recherche, publiés ou non, émanant des établissements d'enseignement et de recherche français ou étrangers, des laboratoires publics ou privés. 


\section{Penser la conception citoyenne de la culture de l'information}

- Version préprint de l'article : Olivier Le Deuff (2009) «La culture de l'information et la dimension citoyenne », Les Cahiers $d u$ Numérique. «La culture informationnelle». Paris HermesLavoisier, vol.5, $\mathrm{n}^{\circ} 3$, p. 39-49

OLIVIER LE DEUFF

OLEDEUFF@GMAIL.COM

ATER. UNIVERSITE DE LYON 3

DOCTORANT EN INFORMATION COMMUNICATION

UNIVERSITE EUROPEENNE DE BRETAGNE

PREFICS.UMR LCF 8143. UNIVERSITE RENNES 2- HAUTE BRETAGNE.

0299941438/0687653127

La culture de l'information constitue à la fois une traduction possible et une vision plus ambitieuse de l'information literacy.

Trois conceptions historiques sont à la base de l'information literacy: celle issue du monde de l'entreprise, celle des bibliothèques notamment universitaires et la «citoyenne», mais ce sont principalement les deux premières qui ont connu le plus de recherche et de travaux. 
La conception économique et celle issue des bibliothèques sont d'ailleurs fortement interconnectées et nullement opposées, notamment dans les articles écrits par des anglophones. Elles se rejoignent ainsi sur plusieurs points en ce qui concerne la nécessité d'impulser de nouvelles méthodes d'apprentissage, notamment en privilégiant des approches par compétences, comme en témoigne le nombre de référentiels et de méthodes tels le Big $6^{1}$ voulant s'appliquer à toute situation.

Les deux conceptions précédentes fonctionnent d'ailleurs en définissant l'information literacy comme une qualité ou compétence personnelle permettant de s'adapter à son environnement informationnel et à la fameuse société de l'information. L'association entre les deux conceptions se remarque ainsi avec les travaux des chercheurs australiens (Lloyd, 2003) qui ont pour objectif de développer le transfert de compétences du monde universitaire à l'entreprise. Du fait que l'information literacy a du mal à gagner pleinement en légitimité universitaire voire disciplinaire, les chercheurs britanniques souhaitent également associer plus nettement la gestion de l'information et la maîtrise de l'information en important des notions issues du management mais également en utilisant des stratégies de gestion de projet (Corrall, 2008) pour développer des programmes de formations. Ces conceptions demeurent fortement inscrites dans un paradigme informationnel qui fait de l'information une matière première.

Nous constatons que la troisième conception de l'information literacy n'a pas été au final très développée, notamment dans l'examen de ses fondements théoriques et historiques qui sont les éléments constitutifs de la culture de l'information. Afin de mieux appréhender cette dimension citoyenne, un retour sur la période des Lumières et le projet encyclopédique, où Brigitte Juanals (Juanals, 2003) voit éclore une culture de l'information, s'impoe. Nous examinerons particulièrement l'état de majorité défini par Emmanuel Kant, que nous considérons comme une aptitude de base pour la constitution de la culture de l'information. Nous nous appuierons particulièrement sur les éclaircissements apportés par Bernard Stiegler (Stiegler, 2008) pour comprendre l'articulation entre Kant et Gilbert Simondon, qui préconise un état de majorité vis-à-vis de la technique. Nous souhaitons montrer ainsi que la conception citoyenne de la culture de

\footnotetext{
${ }^{1}$ M. B. Eisenberg et R. E. Berkowitz (1990). Information Problem Solving: The Big Six Skills Approach to Library \& Information Skills Instruction. Ablex Publishing Corporation
} 
l'information peut être réalisée par le développement d'une culture technique.

\section{La sortie du paradigme informationnel}

Si nous reprenons l'histoire de l'usage du terme d'information literacy, nous pouvons distinguer trois différentes conceptions (Le Deuff, 2007). Ces trois conceptions ne sont pas évidemment exclusives, il faut plutôt les considérer comme des dominantes même s'il s'agit parfois de manières différentes d'envisager l'information.

- La conception économique : le terme information literacy a été utilisé pour la première fois par Paul Zurkowski, président de l'Information Industry Association (IIA) en 1974. Ce dernier souhaitant que les employés soient mieux formés au bon usage et à la bonne gestion de l'information.

- La conception issue des bibliothèques : c'est de loin la plus connue, elle représente l'essentiel des publications. Elle est notamment issue du texte de l'ALA (American Library Association) de 1989. Elle constitue simplement le prolongement de l'instruction bibliographique et son élargissement à l'information disponible sur les réseaux.

- La conception citoyenne : cette conception peut être située en 1976 avec les propos du bibliothécaire Major R. Owens qui devint membre du Congrès et qui faisait de l'information literacy la garantie de la pérennité des institutions démocratiques. Il évoquait notamment la nécessité pour le citoyen de disposer de ressources pertinentes :

"L'information literacy est nécessaire pour garantir la survie des institutions démocratiques. Tous les hommes sont créés égaux, mais les électeurs avec des ressources d'information sont en mesure de prendre des décisions plus intelligentes que les citoyens qui sont des «illettrés informationnels ». La prise en compte de ressources d'informations dans le processus de prise de décision pour l'exercice de pleines responsabilités civiques est une nécessité vitale. »(Owens, 1976, 27)

La conception citoyenne n'a pas connu de réels développements au sein de l'information literacy, si nous exceptons l'article de Shapiro et de Hugues (Shapiro, 1996) qui prônait de nouvelles Lumières en la matière. La dimension citoyenne se trouve fréquemment sous-jacente dans les deux 
conceptions précédentes. Cependant, elles privilégient plutôt un paradigme informationnel et la nécessité de s'adapter à la société de l'information.

\section{Les rapports avec la société de l'information}

L'usage fréquent de l'expression de société de l'information dans les articles sur l'information literacy peut s'expliquer par la valeur conférée à l'information qui devient de fait l'équivalent de la matière première dans une prétendue société de l'information. (Bell, 1974)

Les origines ici sont évidemment communes entre les deux termes puisqu'il s'agit du constat de la valeur économique de l'information et de l'enjeu de former des salariés capables justement d'en tirer le maximum en termes de retour sur investissements. L'ouvrage de Christina Doyle en 1994 (Doyle, 1994) illustre bien cette vision et ce parallèle qui fait souvent de l'information literacy, le pré-requis de la société de l'information. Stratégiquement, la mise en parallèle des deux termes constituait également un moyen de gagner en légitimité et permettait de mieux faire comprendre les objectifs de l'information literacy.

Cette nécessité d'asseoir une légitimité économique de l'information literacy est en fait déjà présente dans le texte de 1989 de l'American Library Association. (Ala, 1989). Il nous semble que la tendance est trop souvent d'oublier que ce texte fut écrit par des américains pour des citoyens américains. Le texte dans sa globalité est souvent oublié et ne demeure plus que la célèbre définition de l'information literacy qui est sans cesse réutilisée :

"To be information literate, a person must be able to recognize when information is needed and have the ability to locate, evaluate, and use effectively the needed information."

Il convient pourtant de refaire une lecture attentive du texte de l'ALA dans son ensemble. Le texte évoque une société de l'information américaine et met l'accent sur les nouvelles nécessités de formation à l'information. L'article repose principalement sur des exemples de situations américaines, notamment d'entreprises qui auraient pu réaliser des économies avec une meilleure gestion de l'information disponible. Le contexte est donc celui d'une défense des bibliothèques et des divers rôles qu'elles peuvent jouer dans la société américaine. Cela concerne aussi bien l'éducation, l'intégration des citoyens et notamment des nouveaux arrivants, mais aussi les qualités de gestion de l'information que l'on peut trouver chez les 
bibliothécaires. Le but est de démontrer le rôle de ces derniers, qui peuvent s'avérer fortement utiles pour le monde économique.

Ce manifeste pro information literacy est donc avant tout un moyen d'inscrire les bibliothèques dans une voie "plus moderne» et dans la perspective de sortir d'une série de représentations qui en ferait l'instrument du passé. Il ne faut pas oublier non plus le contexte politique américain, qui vient d'être marqué par huit ans de présidence républicaine de Ronald Reagan, avec un fort désengagement de l'Etat, ce qui a pleinement touché les bibliothèques. Le texte de l'ALA parait dix jours avant la prise de fonction du nouveau président, lui aussi républicain : George Herbert Walker Bush. Il s'agit donc avant tout d'un message voulant rappeler le rôle des bibliothèques au sein de la société américaine. Le texte mentionne d'ailleurs des aspects de fourniture d'informations aux sociétés privées, une initiative qui avait été déjà préconisée sous la présidence de Carter.

Tout cela doit nous inciter à nouveau à être prudent avec le concept d'information literacy tant sa définition de l'ALA est clairement celle d'un contexte socio-économique américain et qu'elle porte avec elle tout une série de visions et de revendications qui ne sont pas nécessairement opérantes en dehors.

Selon nous, il faut sortir du paradigme informationnel et ne pas chercher à associer la culture de l'information à la société de l'information. Il convient davantage de développer justement la troisième conception qui a été quelque peu mise de côté du fait des représentations des deux autres conceptions dominantes.

\section{La culture de l'information en tant que conception citoyenne}

La culture de l'information est avant tout une formation non seulement au sens éducatif mais également dans le sens de transformation et de processus. Elle n'opère donc pas en parallèle d'une prétendue société de l'information. Elle n'est pas dans une logique d'adaptation mais plutôt de création.

Nous avons donc choisi de privilégier le concept de culture de l'information considérant que l'expression culture informationnelle concerne plutôt l'échelon individuel. La dimension citoyenne de la culture de l'information implique une démarche collective. D'autre part, l'expression de culture informationnelle peut être source de confusion du fait de l'usage qu'en fait Jérôme Segal (Segal, 2003) pour montrer que la théorie de l'information et les thèses cybernétiques ont constitué un terreau pour l'émergence de représentations et de discours. 
Pour mieux comprendre la teneur de cette dimension citoyenne à l'œuvre dans la culture de l'information, il convient de rappeler qu'elle repose sur un héritage documentaire. Il ne s'agit pas pour autant d'un moyen de qualifier autrement la documentation. Nous rejoignons d'ailleurs Claude Baltz (Baltz, 1998) sur le point qu'il faut effectivement que la culture de l'information ne soit pas un concept strictement documentaire et encore moins l'apanage des seuls professionnels de la documentation.

$\mathrm{Du}$ fait notamment de cet héritage documentaire, la culture de l'information est également une culture technique. Elle s'inscrit à la fois dans la lignée des outils constitutifs de la pensée et également dans celle des précurseurs de la documentation qui ont imaginé des systèmes et des méthodes rationnelles pour faciliter l'accès au savoir. Sylvie Fayet-Scribe (Fayet-Scribe, 1999) évoquait même la naissance d'une culture de l'information issue des travaux des pionniers comme Paul Otlet ou Suzanne Briet.

Seulement, la culture de l'information ne saurait reposer uniquement sur la bonne pratique d'outils et de méthodes notamment documentaires. Elle implique davantage des dimensions technique et sociale. Finalement, il convient de repartir de la conclusion de l'ouvrage de Brigitte Juanals (Juanals, 2003) pour commencer à penser cette culture de l'information citoyenne :

"Ainsi, l'accès démocratique à l'information et du savoir sur les plans technologique, médiatique, méthodologique et intellectuel, ne pourra-t-il être que le fruit d'un processus d'apprentissage et d'éducation. De plus, cette culture de l'information ne peut se développer que par l'intégration de cet individu au cadre plus large d'une collectivité - le faisant évoluer ainsi de l'individuel au collectif - et par la prise en compte d'une dimension sociale et citoyenne, qui l'amène à apprendre, à s'informer, à traiter et échanger de l'information au sein des réseaux de personnes. »(Juanals, 2003. p.202)

Selon nous, cette dimension citoyenne repose dès lors sur un état de majorité tout autant technique que citoyen que nous allons examiner.

\section{Culture de l'information et majorité}

La dimension citoyenne de la culture de l'information repose sur un état de majorité qui est autant celui de l'entendement que celui d'un rapport maitrisé vis-à-vis de la technique. Pour le comprendre, un retour sur les racines de la culture de l'information apparait nécessaire. Brigitte Juanals avait vu dans le projet encyclopédique un début de culture de l'information avec la volonté de rassembler et de rendre accessibles les savoirs. Gilbert 
Simondon montre que le projet encyclopédique procède surtout d'une rationalisation de savoirs, notamment techniques. Cette rationalisation permet de pleinement réaliser l'accès au savoir notamment par la « sortie » hors du secret préservé par les systèmes d'initiés ou des corporations. :

"L'encyclopédie réalise une universalité de l'initiation, et par là produit une sorte d'éclatement du sens même de l'initiation, le secret de l'universel objectivé garde de la notion de secret le sens positif (perfection de la connaissance, familiarité avec le sacré), mais annihile le caractère négatif (obscurité, moyens d'exclusion par le mystère, connaissance réservée à un petit nombre d'hommes) La technique devient mystère exotérique. » (Simondon, 1989, p.95)

Simondon insiste d'ailleurs sur le fait que l'encyclopédie n'est pas l'ouvrage ou le pamphlet le plus révolutionnaire mais que son caractère essentiel réside dans le fait qu'elle s'inscrit dans une démarche "adulte », c'est-à-dire basée sur une connaissance rationnelle, théorique, scientifique et universelle (Simondon, p.92). Cette connaissance technique permise par le rassemblement d'une communauté d'hommes animés par un même esprit se manifeste notamment par des planches détaillées pouvant satisfaire la curiosité du lecteur en quête d'érudition mais incitant l'esprit éclairé à agir et poursuivre le travail réalisé par ses prédécesseurs.

Cet esprit éclairé correspondait à un idéal dans la lignée des Lumières et marquait ainsi «la sortie de l'homme de sa minorité dont il est lui-même responsable », définition courte des Lumières exprimée par Kant. Nous avons souhaité ainsi réexaminer plus largement le projet des Lumières, notamment à partir de la réflexion d'Emmanuel Kant, qui fait des lumières la sortie vers la majorité, c'est-à-dire, la capacité à penser par soi-même pour devenir un citoyen éclairé.

Cette capacité repose sur la maitrise de techniques essentielles que sont la lecture et l'écriture afin de devenir savants, c'est-à-dire au sens de celui qui peut accéder au savoir. L'essentiel du texte de Kant repose sur le passage pour l'individu d'une minorité dans laquelle il peut se complaire à une majorité qui constitue le réel établissement de son indépendance d'esprit. Ce passage pouvant s'établir notamment grâce à une culture reposant essentiellement sur le livre et via des capacités de lecture et d'écriture.

Il s'agit donc pour nous de repenser ce passage avec les nouvelles techniques qui constituent notre actuel environnement. Si nous pensons en effet que la définition kantienne peut s'appliquer à la culture de l'information, nous pensons qu'il s'agit bien plus qu'une autonomie dans un environnement informationnel. Il ne s'agit donc pas d'un bon usage des outils mais d'une meilleure prise en compte de la technique et notamment de 
l'objet technique en vue de son intégration à la culture. Selon nous, la culture de l'information repose sur une transmission rendue possible par l'objet technique. Ce dernier pouvant être considéré comme un hypomnematon, terme issu du grec ancien et qui désigne étymologiquement une "sousmémoire». Foucault utilisait le concept pour qualifier les supports de mémoire. Stiegler élargit le concept et réhabilite les hypomnemata en tant que mnémotechniques et technologies de l'esprit. D'ailleurs, la culture de l'information est principalement une culture des hypomnemata en constituant pleinement une culture technique.

\section{Culture de l'information et culture technique}

La culture technique repose sur une relation entre les outils et l'individu qui ne peut être seulement une relation d'usage. Elle implique donc une meilleure compréhension des objets techniques. Simondon entrevoit notre relation à la technique au sein de deux états opposés. Nous sommes donc selon lui, soit dans un état de minorité, soit dans un état de majorité face à l'objet technique :

"L'objet technique peut être rattaché à l'homme de deux manières opposées : selon un statut de majorité ou selon un statut de minorité.» (Simondon, 1989, p.85)

Ces deux positions doivent être différenciées des deux écueils que souhaite éviter Simondon : la technophobie et la technophilie, positions qui ne sont que les révélatrices d'une non-intégration de la technique à la culture :

"Les idées d'asservissement et de libération sont beaucoup trop liées à l'ancien statut de l'homme comme objet technique pour pouvoir correspondre au vrai problème de la relation de l'homme et de la machine. Il est nécessaire que l'objet technique soit connu en lui-même pour que la relation de l'homme à la machine devienne stable et valide: d'où la nécessité d'une culture technique. »(Simondon, 1989, p.32)

Cette culture technique suppose une vision d'ensemble et une compréhension globale puisqu'il est évidemment impossible de posséder une connaissance poussée de l'ensemble des objets techniques qui nous entourent.

\section{L'état de minorité.}


Dans l'état minoritaire, la technique n'est justement pas pensée, elle est oubliée voire évacuée tant elle est devenue constitutive de notre environnement :

"Le statut de minorité est celui selon lequel l'objet technique est avant tout un objet d'usage, nécessaire à la vie quotidienne, faisant partie de l'entourage au milieu duquel l'individu humain grandit et se forme.(...) Le savoir technique est implicite, non réfléchi, coutumier. »(Simondon, 1989, p.85)

Il n'y a donc pas de réflexion sur les usages ni prise de distance par rapport à l'objet. Seule la logique purement utilitaire prédomine et il n'y a pas de rationalisation des savoirs.

L'état de minorité est bien sûr initialement détaillé avec Kant dans une opposition avec l'état de majorité qui est celui du citoyen éclairé. Kant insiste en fait davantage sur les caractéristiques de l'état de minorité, ce qui fait d'ailleurs dire à Foucault que ce texte est principalement négatif (Foucault, 1984). Il définit donc d'emblée les Lumières par opposition à l'état de minorité :

"Minorité, c'est-à-dire incapacité de se servir de son entendement (pouvoir de penser) sans la direction d'autrui, minorité dont il est lui-même responsable (faute) puisque la cause en réside non dans un défaut de l'entendement mais dans un manque de décision et de courage de s'en servir sans la direction d'autrui. Sapere aude! (Ose penser) Aie le courage de te servir de ton propre entendement. » (Kant, 1784)

L'état de minorité serait donc ainsi un état de servitude volontaire. Il convient de nuancer ces propos en notant que cette passivité est conditionnée surtout par l'absence de formation. Or cette formation (Bildung) ne peut résider que par une volonté de former à une majorité critique. Cependant les techniques, sur lesquelles pourrait reposer une formation à la majorité, sont le plus souvent utilisées pour faire demeurer les individus dans un état de minorité :

«La plupart du temps, ces techniques visent à contrôler l'attention non pas pour susciter en elle le courage et la volonté de savoir, mais tout au contraire pour la maintenir dans son état de minorité adulte.. » (Stiegler, 2008, p.72)

Quelque part, il nous faut donc enseigner ces techniques, ces hypomnemata, c'est-à-dire « éduquer », conduire à la majorité. L'Aufklärung suppose une prise de distance, une capacité à s'extraire des dogmes, des idées préconçues et véhiculées par d'autres : 
La paresse et la lâcheté sont les causes qui expliquent qu'un si grand nombre d'hommes, après que la nature les a affranchi depuis longtemps d'une (de toute) direction étrangère, reste cependant volontiers, leur vie durant, mineurs, et qu'il soit facile à d'autres de se poser en tuteur des premiers. Il est si aisé d'être mineur! Si j'ai un livre qui me tient lieu d'entendement, un directeur qui me tient lieu de conscience, un médecin qui décide pour moi de mon régime, etc., je n'ai vraiment pas besoin de me donner de peine moi-même. (Kant, 1784)

Nous notons qu'il s'agit donc de produire un effort face à la « facilité ». En cela, la sortie de la minorité nécessite des étapes, des phases afin de pouvoir exercer sa raison :

"J'entends par usage public de notre propre raison celui que l'on en fait comme savant devant l'ensemble du public qui lit. » (Kant, 1784)

La phrase de Kant est quelque peu difficile à interpréter, mais Stiegler nous « éclaire » sur ce point :

"L'écrivain et le public de lecteurs dont parle Kant sont majeurs en cela que lisant, se lisant, et étant susceptibles d'écrire à tout moment ce qu'ils ont lu, soit pour en poursuivre l'écriture, dans le cas de l'écrivain (...) soit pour écrire un autre livre, ou un article, ou un rapport, ou une note de synthèse, ou un commentaire de texte, dans le cas du lecteur (...), ils accèdent les uns, les autres à la forme critique de l'attention. » (Stiegler, 2008, p.48)

L'accès ne concerne pas seulement l'information en tant que telle mais plutôt la capacité à pouvoir la critiquer. Nous serions tentés de rajouter à la liste de Stiegler, le terme de billet de blog tant ces techniques de base demeurent essentielles dans les technologies actuelles et autre hypomnemata numériques.

\section{Conclusion}

La culture de l'information en tant que conception citoyenne permet davantage de penser justement la formation des individus dans une relation qui repose sur une culture technique. Les outils du numérique nécessitent des savoirs et des savoir-faire qui dépassent le simple usage que des dispositifs comme le B2I tente parfois de mesurer. En effet, il s'agit d'éviter la confusion entre compétences informationnelles et capacités intellectuelles, ce qui risquerait d'accélérer ce que Bernard Stiegler nomme «la liquidation 
de la faculté cognitive, qui est remplacée par l'habileté informationnelle »( Stiegler, 2008, p.48)

La dimension citoyenne repose sur une prise en compte des hypomnemata qui sont des pharmaka, c'est-à-dire qu'ils permettent autant la constitution de la culture de l'information qu'ils peuvent en constituer des obstacles. C'est ici que s'opère alors, la capacité à se servir de son entendement telle que le recommande Kant, en étant capable d'utiliser les outils de manière à mieux comprendre un problème, une situation afin de pouvoir agir de manière consciente. Sans cette capacité d'attention et de distance critique, d'autres peuvent utiliser ces outils pour justement priver l'individu de son entendement comme cela peut être le cas en ce qui concerne les stratégies publicitaires.

Cette compréhension des hypomnemata ne peut être que le résultat d'une formation et d'une éducation. C'est pleinement le rôle de transmission de la culture de l'information, rôle à la fois intergénérationnel et éminemment culturel :

"La culture n'est rien d'autre que la capacité d'hériter collectivement de l'expérience de nos ancêtres et cela a été compris depuis longtemps. Ce qui a été moins compris, c'est que la technique (...) est la condition d'une telle transmission. » (Stiegler, 1998, p.193)

\section{Bibliographie}

American Library Association Presidential Committee on Information literacy., Final Report, Chicago:American Library Association, 1989

Baltz, C.,. «Une culture pour la société de l'information ? Position théorique, définition, enjeux » Documentaliste, Vol.35 n², 1998, p.75-82. p.82

Bell, D.,. Vers une société post-industrielle, Robert Laffont, 1974

Corral, S., «Information literacy strategy development in higher education: An exploratory study », International Journal of Information Management, 2008, 28, no. 1 p. 26-37.

Doyle, C., Information literacy in an Information Society. A concept for the information age, ERIC \& Clearinghouse on Information \& Technology, Syracuse University, 1994 
Foucault, M., «What is Enligthenment ? «(«Qu'est-ce que les Lumières? «), in Rabinow P., éd., The Foucault Reader, New York, Pantheon Books, 1984, p. 32-50.

Sylvie, Fayet-Scribe. Histoire des outils de médiation du savoir, naissance d'une culture de l'information 1895-1937. Thèse d'habilitation à diriger des recherches, Paris 1, 1999

Kant, Emmanuel. «Beantwortung der Frage : Was ist Aufklärung ?», Berlinische Monaesschrift, 4, 1784, 481-494, trad. Jules BARNI. Réponse à cette question : «Qu'est-ce que les Lumières?» disp. sur : <http://fr.wikisource.org/wiki/Qu\%E2\%80\%99estce_que_les_Lumi\%C3\%A8res_\%3F>

Le Deuff, O., «La culture de l'information: Quelles « littératies » pour quelles conceptions de l'information?» in VI.ème Colloque ISKO-France'2007 7 et 8 juin 2007, Toulouse, IUT de l'Université Paul Sabatier

Lloyd, A., Information literacy: «The meta-competency of the knowledge economy? an exploratory paper. », Journal of Librarianship and Information Science, 2003, 35(2), 87-92.

Owens, M., « State Government and Libraries. » Library Journal 101, 1976, p.27

Serres, A.,. «La culture informationnelle». In Papy, Fabrice (sous la dir. de). Problématiques émergentes dans les sciences de l'information. Paris : Hermès Lavoisier, 2008, p.137-160.

Segal, J., «Statut implicite de la notion d'information dans quelques théories biologiques et philosophiques, conséquences épistémologiques », Séminaires de recherche en épistémologie de la communication, de l'information et de la cognition de la Télé-Université de l'Université du Quebec, Montréal, 2005. Disp. sur <http://jerome-segal.de/Publis/Alliage.htm

Shapiro, J.,Hugues, S. K.., «Information literacy as a Liberal Art : Enlightenment Proposals for a New Curriculum. »In Educom Review. 1996 n $^{\circ} 31$, mars-avril Disp. Sur : <http://net.educause.edu/apps/er/review/reviewArticles/31231.html>

Simondon, G., Du mode d'existence des objets techniques, Paris, Aubier, 1989

Stiegler, B., Prendre soin : Tome 1, De la jeunesse et des générations, Flammarion, 2008

Stiegler, B., « Leroi-Gourhan : l'inorganique organisé » Les Cahiers de médiologie, 1998, n6, 1 p. 189.p.193 